\title{
ON THE CATEGORIES OF PARAGRADED GROUPS AND MODULES OF TYPE $\Delta$
}

\author{
EMIL ILIĆ-GEORGIJEVIĆ
}

\begin{abstract}
In this paper we observe the categories of paragraded groups and $R$-modules with respect to the same set of grades $\Delta$, where $R$ is the paragraded ring with the set of grades $\Delta$. We turn our attention to constructing new objects in those categories using the sets of morphisms of grade $\delta \in \Delta$. This process defines bifunctors which happen to be left exact. Thus we may construct the right derived functor and it turns out that it behaves the same as in the case of the category of abstract modules.
\end{abstract}

\section{INTRODUCTION}

Paragraded groups were introduced by M. Krasner and M. Vuković ([7]) in order to solve a problem of graded groups: they are not closed with respect to the direct product in sense that the homogeneous part of the direct product is not the direct product of the homogeneous parts of its components. Actually, from the homogeneous point of view, a multigroupoid does not have to be a homogroupoid ([7]). Paragraded groups are defined in [7] by the six-axiom system as follows.

Definition 1.1. The map $\pi: \Delta \rightarrow \operatorname{Sg}(G), \pi(\delta)=G_{\delta}(\delta \in \Delta)$, of a partially ordered set $(\Delta,<)$, which is from bellow a complete semi-lattice and from above inductively ordered, to the set $\operatorname{Sg}(G)$ of subgroups of the group $G$, is called a paragraduation if it satisfies the following six-axiom system:

i) $\pi(0)=G_{0}=\{e\}$, where $0=\inf \Delta ; \delta<\delta^{\prime} \Rightarrow G_{\delta} \subseteq G_{\delta^{\prime}}$;

Remark 1.2. $H=\bigcup_{\delta \in \Delta} G_{\delta}$ is called the homogeneous part of $G$ with respect to $\pi$, and elements from $H$ are called the homogeneous elements of $G$.

2000 Mathematics Subject Classification. 20J99, 16D90, 16W99, 18A22.

Key words and phrases. Category, paragraded group, paragraded module, a morphism of grade $\delta$, left exact bifunctor $\operatorname{HOM}(-,-)$. 
Remark 1.3. If $x \in H$, we say that $\delta(x)=\inf \left\{\delta \in \Delta \mid x \in G_{\delta}\right\}$ is a grade of $x$. We have $\delta(x)=0$ iff $x=e$. The elements $\delta(x), x \in H$, are called the principal grades and they form a set which we will denote by $\Delta_{p}$.

ii) $\theta \subseteq \Delta \Rightarrow \bigcap_{\delta \in \theta} G_{\delta}=G_{\inf \theta}$;

iii) If $x, y \in H$ and $y x=z x y$, then $z \in H$ and $\delta(z) \leq \inf (\delta(x), \delta(y))$;

iv) The homogeneous part $H$ is a generating set of $G$;

v) Let $A \subseteq H$ be a subset such that for all $x, y \in A$ there exists an upper bound for $\delta(x), \delta(y)$. Then there exists an upper bound for all $\delta(x), x \in A$

vi) $G$ is generated by $H$ with the set of $H$-internal and left commutation relations (see [7]).

The group is called paragraded if it has a paragraduation.

If we replace the sixth axiom with the following axiom:

vi') Let $\delta_{1}, \ldots, \delta_{s} \in \Delta_{p}$ be pairwise incomparable and let $x_{i}, x_{i}^{\prime} \in H$ $(i=1, \ldots, s)$ be such that $x_{1} \cdots x_{s}=x_{1}^{\prime} \cdots x_{s}^{\prime}$ and $x_{i}, x_{i}^{\prime} \in G_{\delta_{i}}$ for all $i=1, \ldots, s$. Then $\delta\left(x_{i}^{-1} x_{i}^{\prime}\right)<\delta_{i}$,

we get the notion of an extragraded group.

Theorem 1.4 ([7]). Every extragraded group is a paragraded group.

We shall consider maps between paragraded groups as well.

Let $G_{1}$ and $G_{2}$ be two paragraded groups with sets of grades $\Delta_{1}$ and $\Delta_{2}$, paragraduations $\pi_{1}$ and $\pi_{2}$, and homogeneous parts $H_{1}$ and $H_{2}$ respectively.

Definition 1.5. ([7]) We say that a homomorphism $f: G_{1} \rightarrow G_{2}$ is a quasihomogeneous if

$$
\left(\forall x \in H_{1}\right) f(x) \in H_{2} .
$$

As we will see, we shall confine ourselves to the case $\Delta_{1}=\Delta_{2}$.

Definition 1.6. ([7]) The ring $(R,+, \cdot)$ is called paragraded if its additive group $(R,+)$ is a paragraded group, with paragraduation $\pi$ and set of grades $\Delta$, and if

$$
(\forall \xi, \eta \in \Delta)(\exists \zeta \in \Delta) R_{\xi} R_{\eta} \subseteq R_{\zeta} .
$$

Definition 1.7. ([7]) If $R$ is a paragraded ring with paragraduation $\pi$, then the map $(\xi, \eta) \rightarrow \xi \eta$ from $\Delta \times \Delta$ to $\Delta$ is called $\Delta$-multiplication of grades if the following holds:

a) $R_{\xi} R_{\eta} \subseteq R_{\xi \eta}$

b) $\left(\forall \xi, \xi^{\prime}, \eta, \eta^{\prime} \in \Delta\right) \xi \leq \xi^{\prime} \wedge \eta \leq \eta^{\prime} \Rightarrow \xi \eta \leq \xi^{\prime} \eta^{\prime}$. 
If $R$ is a paragraded ring with paragraduation $\pi$, then there exists $\zeta=$ $\sup \left(\delta(z) \mid z \in R_{\xi} R_{\eta}\right)$. If we put $\zeta=\xi \eta$, we will get $\Delta$-multiplication and we call it minimal multiplication $([7])$.

Definition 1.8. ([7]) Let $R_{1}$ and $R_{2}$ be two paragraded rings and $f: R_{1} \rightarrow$ $R_{2}$ a homomorphism. We say that this homomorphism is a quasihomogeneous if it is a quasihomogeneous homomorphism from a paragraded group $\left(R_{1},+\right)$ to a paragraded group $\left(R_{2},+\right)$.

Now, we will give the definition of a paragraded module.

Definition 1.9. ([7]) Let $R$ be a paragraded ring with paragraduation $E$ and set of grades $\Delta, M$ a commutative paragraded group with paragraduation $F$ and set of grades $D$ and suppose $M$ is an $R$-module. Denote $E(\delta)$ by $R_{\delta}$ and $F(d)$ by $M_{d}$, where $\delta \in \Delta, d \in D$. The R-module $M$ is called paragraded if

$$
(\forall \delta \in \Delta)(\forall d \in D)(\exists t \in D) R_{\delta} M_{d} \subseteq M_{t} .
$$

Definition 1.10. ([7]) The map $\Delta \times D \rightarrow D:(\delta, d) \rightarrow \delta d$ is called $(\Delta, D)$ multiplication if:

1. $R_{\delta} M_{d} \subseteq M_{\delta d}$;

2. $\left(\forall \delta, \delta^{\prime} \in \Delta\right)\left(\forall d, d^{\prime} \in D\right) \delta \leq \delta^{\prime} \wedge d \leq d^{\prime} \Rightarrow \delta d \leq \delta^{\prime} d^{\prime}$.

It is always possible to construct $(\Delta, D)$-multiplication by putting $\delta d=$ $\sup _{z \in R_{\delta} M_{d}} d(z)([7])$. This multiplication is called minimal multiplication.

The main feature of paragraded structures is described in the following theorem.

Theorem 1.11 ([7]). The direct product of paragraded structures (groups, rings and modules) is also a paragraded structure and the homogeneous part of direct product is the direct product of the homogeneous parts of the components.

\section{The CATEgories $G_{\Delta}^{P}$ And $M_{\Delta}^{P}$}

Let us observe the category of paragraded groups whose set of grades is $\Delta$ and denote it by $G_{\Delta}^{P}$. We call it the category of paragraded groups of type $\Delta$. Objects of such a category are paragraded groups, and morphisms are the elements of the set

$$
\operatorname{hom}_{G_{\Delta}^{P}}\left(G, G^{\prime}\right)=\left\{f \in \operatorname{hom}\left(G, G^{\prime}\right) \mid f\left(G_{\delta}\right) \subseteq G_{\delta}^{\prime}, \delta \in \Delta\right\},
$$

where $G, G^{\prime}$ are paragraded groups.

Let us observe the paragraded $R$-modules $M$, where $R$ is a paragraded ring with set of grades $\Delta$ and $M$ is a commutative paragraded group of type $\Delta$. These modules together with the set of morphisms

$$
\left\{f \in \operatorname{hom}\left(M, M^{\prime}\right) \mid f\left(M_{\delta}\right) \subseteq M_{\delta}^{\prime}, \delta \in \Delta\right\}
$$


form a category which we will denote by $M_{\Delta}^{P}$. We call it the category of paragraded $R$-modules of type $\Delta$.

If $\Delta^{\prime} \subseteq \Delta$ and if $\pi$ is the paragraduation of $G$, then $\pi^{\prime}=\left.\pi\right|_{\Delta^{\prime}}$ is also a paragraduation if $\Delta^{\prime}$ is as ordered as $\Delta$. So, if that is the case, we may observe the category $G_{\Delta^{\prime}}^{P}$. Next, we examine the nature of the functor $F$ : $G_{\Delta}^{P} \rightarrow G_{\Delta^{\prime}}^{P}$

Proposition 2.1. The functor $F: G_{\Delta}^{P} \rightarrow G_{\Delta^{\prime}}^{P}$ has a right adjoint.

Proof. Let $G^{\prime} \in G_{\Delta^{\prime}}^{P}$ and let $G$ and $G^{\prime}$ be equal as abstract groups, $\pi(\delta)=$ $\pi^{\prime}(\delta)$ for $\delta \in \Delta^{\prime}$ and $\pi(\delta)=\{e\}$ for $\delta \notin \Delta^{\prime}$. Thus, we defined the functor $U$ on objects. Now, we define it on morphisms. If $\varphi^{\prime} \in \operatorname{hom}_{G_{\Delta^{\prime}}^{P}}\left(G_{1}^{\prime}, G_{2}^{\prime}\right)$, then let $U\left(\varphi^{\prime}\right)=\varphi$ be a quasihomogeneous homomorphism defined via $\varphi(x)=\varphi^{\prime}(x)$ if $x \in H^{\prime}$ and $\varphi(x)=e$ otherwise, where $H^{\prime}=\bigcup_{\delta \in \Delta^{\prime}} G_{\delta}$. This functor is the right adjoint. Indeed, for $G \in G_{\Delta}^{P}$ and $G^{\prime} \in G_{\Delta^{\prime}}^{P}$, define a map

$$
f: \operatorname{hom}_{G_{\Delta^{\prime}}^{P}}\left(F(G), G^{\prime}\right) \rightarrow \operatorname{hom}_{G_{\Delta}^{P}}\left(G, U\left(G^{\prime}\right)\right)
$$

by $f(\varphi)(x)=\varphi(x)$ if $x \in H^{\prime}$, and $f(\varphi)(x)=e$ if $x \notin H^{\prime}$, for any $\varphi \in$ $\operatorname{hom}_{G_{\Delta^{\prime}}^{P}}\left(F(G), G^{\prime}\right)$. One easily verifies that for all $g:\left(G, G^{\prime}\right) \rightarrow\left(G_{1}, G_{1}^{\prime}\right)$ the following diagram commutes

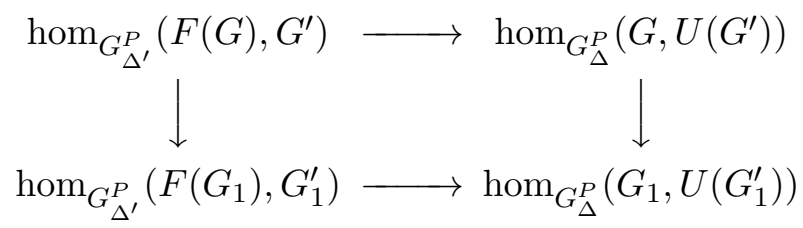

and that $f$ is a bijection, i.e. $f$ is a natural isomorphism.

Proposition 2.2. The category $M_{\Delta}^{P}$ is abelian.

Proof. We will only check whether the category $M_{\Delta}^{P}$ has products and coproducts, since all other axioms are trivial.

Let $M$ and $M^{\prime}$ be paragraded modules of type $\Delta$. We claim that $M \oplus M^{\prime}$ is their coproduct in the category $M_{\Delta}^{P}$. For $\delta \in \Delta$ define $\pi: \Delta \rightarrow \operatorname{Sg}\left(M \oplus M^{\prime}\right)$ by

$$
\pi(\delta)=M_{\delta} \oplus M_{\delta}^{\prime} .
$$

Since in our case we have the same set of grades $\Delta$ of paragraduations, it is the special case of the Theorem 1.11 and so, $\pi$ is the paragraduation of $M \oplus M^{\prime}$. Now, observe the maps $\alpha: M \rightarrow M \oplus M^{\prime}$ and $\beta: M^{\prime} \rightarrow$ $M \oplus M^{\prime}$ defined by $\alpha(m)=(m, 0)$ and $\beta\left(m^{\prime}\right)=\left(0, m^{\prime}\right)$ for all $m \in M$ and $m^{\prime} \in M^{\prime}$. One can easily verify that the maps $\alpha$ and $\beta$ belong to $\operatorname{hom}_{M_{\Delta}^{P}}\left(M, M \oplus M^{\prime}\right)$ and $\operatorname{hom}_{M_{\Delta}^{P}}\left(M^{\prime}, M \oplus M^{\prime}\right)$, respectively. Let $X \in M_{\Delta}^{P}$ 
and $f \in \operatorname{hom}_{M_{\Delta}^{P}}(M, X), g \in \operatorname{hom}_{M_{\Delta}^{P}}\left(M^{\prime}, X\right)$. Define $\theta: M \oplus M^{\prime} \rightarrow X$ by $\theta\left(m, m^{\prime}\right)=f(m)+g\left(m^{\prime}\right)$. If $\delta \in \Delta$, then we have

$$
\theta(\pi(\delta))=\theta\left(M_{\delta} \oplus M_{\delta}^{\prime}\right)=f\left(M_{\delta}\right)+g\left(M_{\delta}^{\prime}\right) \subseteq X_{\delta}+X_{\delta}=X_{\delta} .
$$

Hence,

$$
\theta \in \operatorname{hom}_{M_{\Delta}^{P}}\left(M \oplus M^{\prime}, X\right) .
$$

We have $\theta \alpha(m)=\theta(m, 0)=f(m)$ and $\theta \beta\left(m^{\prime}\right)=\theta\left(0, m^{\prime}\right)=g\left(m^{\prime}\right)$. If $\tau: M \oplus M^{\prime} \rightarrow X$ is a morphism such that $\tau \alpha=f$ and $\tau \beta=g$, then $\tau(m, 0)=f(m)$ for all $m \in M$ and $\tau\left(0, m^{\prime}\right)=g\left(m^{\prime}\right)$ for all $m^{\prime} \in M^{\prime}$. One can easily prove that $\tau\left(m, m^{\prime}\right)=f(m)+g\left(m^{\prime}\right)$ and therefore, $\tau=\theta$.

Analogously, we can prove that the category $M_{\Delta}^{P}$ has products.

Corollary 2.3. The category of abelian paragraded groups of type $\Delta$ is abelian.

Let $G, G^{\prime} \in G_{\Delta}^{P}$ and $M, M^{\prime} \in M_{\Delta}^{P}$.

Definition 2.4. For a homomorphism $f: G \rightarrow G^{\prime}$ we say that it is a morphism of grade $\delta$ if

$$
\left(\forall \delta^{\prime} \in \Delta\right) f\left(G_{\delta^{\prime}}\right) \subseteq G_{\delta}^{\prime} .
$$

For a homomorphism $f: M \rightarrow M^{\prime}$ we say that it is a morphism of grade $\delta$ if

$$
\left(\forall \delta^{\prime} \in \Delta\right) f\left(M_{\delta^{\prime}}\right) \subseteq M_{\delta^{\prime} \delta}^{\prime},
$$

where $\delta^{\prime} \delta$ is minimal multiplication.

Let us denote the set of all the morphisms of grade $\delta$ by $\operatorname{hom}\left(G, G^{\prime}\right)_{\delta}$ and by $\operatorname{hom}\left(M, M^{\prime}\right)_{\delta}$, respectively.

Lemma 2.5. Let $G, G^{\prime}$ be commutative paragraded groups of type $\Delta$ and let $M, M^{\prime}$ be paragraded $R$-modules of type $\Delta$. Then:

a) The set $\operatorname{hom}\left(G, G^{\prime}\right)_{\delta}$ is the subgroup of $\operatorname{hom}\left(G, G^{\prime}\right)$;

b) The set $\operatorname{hom}\left(M, M^{\prime}\right)_{\delta}$ is the subgroup of $\operatorname{hom}\left(M, M^{\prime}\right)$.

Proof. a) Let $f, g \in \operatorname{hom}\left(G, G^{\prime}\right)_{\delta}$. Then,

$$
\left(\forall \delta^{\prime} \in \Delta\right) f\left(G_{\delta^{\prime}}\right) \subseteq G_{\delta}^{\prime} \wedge g\left(G_{\delta^{\prime}}\right) \subseteq G_{\delta}^{\prime},
$$

and hence, $f \cdot g\left(G_{\delta^{\prime}}\right)=f\left(G_{\delta^{\prime}}\right) g\left(G_{\delta^{\prime}}\right) \subseteq G_{\delta}^{\prime} G_{\delta}^{\prime}=G_{\delta}^{\prime}$ and $f^{-1}\left(G_{\delta^{\prime}}\right)=$ $\left(f\left(G_{\delta^{\prime}}\right)\right)^{-1} \subseteq\left(G_{\delta}^{\prime}\right)^{-1}=G_{\delta}^{\prime}$. So, $f g$ and $f^{-1}$ belong to $\operatorname{hom}\left(G, G^{\prime}\right)_{\delta}$, hence, $\operatorname{hom}\left(G, G^{\prime}\right)_{\delta}<\operatorname{hom}\left(G, G^{\prime}\right)$.

b) Let $f, g \in \operatorname{hom}\left(M, M^{\prime}\right)_{\delta}$. Then,

$$
\left(\forall \delta^{\prime} \in \Delta\right) f\left(M_{\delta^{\prime}}\right) \subseteq M_{\delta^{\prime} \delta}^{\prime} \wedge g\left(M_{\delta^{\prime}}\right) \subseteq M_{\delta^{\prime} \delta}^{\prime},
$$


and hence, $f+g\left(M_{\delta^{\prime}}\right)=f\left(M_{\delta^{\prime}}\right)+g\left(M_{\delta^{\prime}}\right) \subseteq M_{\delta^{\prime} \delta}^{\prime}+M_{\delta^{\prime} \delta}^{\prime}=M_{\delta^{\prime} \delta}^{\prime}$ and $-f\left(M_{\delta^{\prime}}\right) \subseteq\left(-M_{\delta^{\prime} \delta}^{\prime}\right)=M_{\delta^{\prime} \delta}^{\prime}$. So, $f+g$ and $-f$ belong to $\operatorname{hom}\left(M, M^{\prime}\right)_{\delta}$, hence, $\operatorname{hom}\left(M, M^{\prime}\right)_{\delta}<\operatorname{hom}\left(M, M^{\prime}\right)$.

Now, consider the sets

$$
\begin{aligned}
\operatorname{HOM}\left(G, G^{\prime}\right) & =\left\langle\bigcup_{\delta \in \Delta} \operatorname{hom}\left(G, G^{\prime}\right)_{\delta}\right\rangle \quad \text { and } \\
\operatorname{HOM}\left(M, M^{\prime}\right) & =\left\langle\bigcup_{\delta \in \Delta} \operatorname{hom}\left(M, M^{\prime}\right)_{\delta}\right\rangle .
\end{aligned}
$$

Theorem 2.6. Let $G$ and $M, M^{\prime}$ be a commutative paragraded group and paragraded $R$-modules of type $\Delta$, respectively, and let $G^{\prime}$ be a commutative extragraded group of type $\Delta$. Then

a) $\operatorname{HOM}\left(G, G^{\prime}\right)$ is the commutative paragraded group of type $\Delta$;

b) $\operatorname{HOM}\left(M, M^{\prime}\right)$ is the commutative paragraded group of type $\Delta$.

Proof. a) According to the previous Lemma, it is easy to establish that $\operatorname{HOM}\left(G, G^{\prime}\right)$ is a group. Now we set off to prove that it is paragraded. Define the map

$$
\pi: \Delta \rightarrow \operatorname{Sg}\left(\operatorname{HOM}\left(G, G^{\prime}\right)\right)
$$

by

$$
\pi(\delta)=\operatorname{hom}\left(G, G^{\prime}\right)_{\delta} .
$$

We first need to consider $\pi(0)$ i.e. the set $\operatorname{hom}\left(G, G^{\prime}\right)_{0}$. It is the set of all morphisms $f: G \rightarrow G^{\prime}$ such that $f\left(G_{\delta^{\prime}}\right) \subseteq G_{0}^{\prime}$ for all $\delta^{\prime} \in \Delta$. We know (see $i)$ ) that $G_{0}^{\prime}=\{e\}$, and hence $f\left(G_{\delta^{\prime}}\right)=\{e\}$ for all $\delta^{\prime} \in \Delta$, i.e. $\operatorname{hom}\left(G, G^{\prime}\right)_{0}=\left\{f_{0}\right\}$, where by $f_{0}$ we denoted the map $g \rightarrow e(g \in G)$. Now, let $\delta_{1}<\delta_{2}$. Then, $\pi\left(\delta_{1}\right)=\operatorname{hom}\left(G, G^{\prime}\right)_{\delta_{1}}$ and $\pi\left(\delta_{2}\right)=\operatorname{hom}\left(G, G^{\prime}\right)_{\delta_{2}}$. Take $f \in \operatorname{hom}\left(G, G^{\prime}\right)_{\delta_{1}}$. Then, for all $\delta^{\prime} \in \Delta$, we have $f\left(G_{\delta^{\prime}}\right) \subseteq G_{\delta_{1}}^{\prime}$. From $\delta_{1}<\delta_{2}$ it follows that $G_{\delta_{1}}^{\prime} \subseteq G_{\delta_{2}}^{\prime}$, according to $i$ ). Hence, $f\left(G_{\delta^{\prime}}\right) \subseteq G_{\delta_{2}}^{\prime}$ for all $\delta^{\prime} \in \Delta$, so $f \in \operatorname{hom}\left(G, G^{\prime}\right)_{\delta_{2}}$ and $\operatorname{hom}\left(G, G^{\prime}\right)_{\delta_{1}} \subseteq \operatorname{hom}\left(G, G^{\prime}\right)_{\delta_{2}}$.

Let us now consider the subset $\theta \subset \Delta$. We wonder what $\bigcap_{\delta \in \theta} \operatorname{hom}\left(G, G^{\prime}\right)_{\delta}$ is. If $f \in \bigcap_{\delta \in \theta} \operatorname{hom}\left(G, G^{\prime}\right)_{\delta}$, then $f\left(G_{\delta^{\prime}}\right) \subseteq G_{\delta}^{\prime}$ for all $\delta^{\prime} \in \Delta$ and for all $\delta \in \theta$. Hence, $f\left(G_{\delta^{\prime}}\right) \subseteq \bigcap_{\delta \in \theta} G_{\delta}^{\prime}$ for all $\delta^{\prime} \in \Delta$ i.e. $f\left(G_{\delta^{\prime}}\right) \subseteq G_{\text {inf } \theta}^{\prime}$ for all $\delta^{\prime} \in \Delta$ according to $\left.i i\right)$. Thus, $\bigcap_{\delta \in \theta} \operatorname{hom}\left(G, G^{\prime}\right)_{\delta}=\operatorname{hom}\left(G, G^{\prime}\right)_{\inf \theta}$.

Denote by $H$ the set $\bigcup_{\delta \in \Delta} \operatorname{hom}\left(G, G^{\prime}\right)_{\delta}$. Take two elements $f, g \in H$. Then there are $\delta_{1}$ and $\delta_{2}$ from $\Delta$ such that $f \in \operatorname{hom}\left(G, G^{\prime}\right)_{\delta_{1}}$ and $g \in \operatorname{hom}\left(G, G^{\prime}\right)_{\delta_{2}}$. Let $h$ be $g f g^{-1} f^{-1}$. We wish to prove that $h \in H$. For all $\delta^{\prime} \in \Delta$ we have

$$
\begin{aligned}
h\left(G_{\delta^{\prime}}\right) & =g\left(G_{\delta^{\prime}}\right) f\left(G_{\delta^{\prime}}\right) g^{-1}\left(G_{\delta^{\prime}}\right) f^{-1}\left(G_{\delta^{\prime}}\right) \\
& \subseteq G_{\delta_{2}}^{\prime} G_{\delta_{1}}^{\prime}\left(G_{\delta_{2}}^{\prime}\right)^{-1}\left(G_{\delta_{1}}^{\prime}\right)^{-1} .
\end{aligned}
$$


We will now show how we can avoid the commutativity of $G^{\prime}$ in this case. Since $G_{\delta_{1}}^{\prime}$ and $G_{\delta_{2}}^{\prime}$ are normal subgroups (see [7]), it follows that

$$
h\left(G_{\delta^{\prime}}\right) \subseteq G_{\delta_{1}}^{\prime} \cap G_{\delta_{2}}^{\prime} \stackrel{i i)}{=} G_{\mathrm{inf}\left(\delta_{1}, \delta_{2}\right)}^{\prime}\left(\delta^{\prime} \in \Delta\right) .
$$

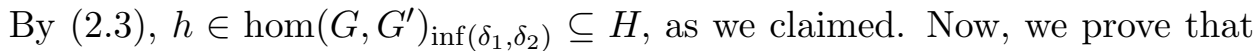
$\delta(h) \leq \inf (\delta(f), \delta(g))$. But this follows from the fact that $\delta(h) \leq \inf \left(\delta_{1}, \delta_{2}\right)$.

The set $\operatorname{HOM}\left(G, G^{\prime}\right)$ is generated by $H$ as it can be seen from the construction of the set $\operatorname{HOM}\left(G, G^{\prime}\right)$.

Assume now that we have a subset $A \subseteq H$ such that for all $f, g \in A$ there exists $\delta \in \Delta$ such that $f g \in \operatorname{hom}\left(G, G^{\prime}\right)_{\delta}$. We wish to prove that there exists $\delta_{1} \in \Delta$ such that $A \subseteq \operatorname{hom}\left(G, G^{\prime}\right)_{\delta_{1}}$. For any $\delta^{\prime} \in \Delta$ choose $x \in G_{\delta^{\prime}}$. Then $f(x) g(x) \in H_{G^{\prime}}$ and so by $\left.v\right), f(x) \in G_{\delta_{1}}^{\prime}$ for some $\delta_{1} \in \Delta$. The map $f$ was arbitrary, so $A \subseteq \operatorname{hom}\left(G, G^{\prime}\right)_{\delta_{1}}$ as we wished to prove.

Let $\delta_{1}, \ldots, \delta_{s} \in \Delta_{p}$ be mutually incomparable and $f_{1} \ldots f_{s}=f_{1}^{\prime} \ldots f_{s}^{\prime}$, where $f_{i}, f_{i}^{\prime} \in \operatorname{hom}\left(G, G^{\prime}\right)_{\delta_{i}}(i=\overline{1, s})$. That means that for arbitrarily chosen $x \in H_{G}$ one has

$$
f_{1}(x) \ldots f_{s}(x)=f_{1}^{\prime}(x) \ldots f_{s}^{\prime}(x)
$$

and $f_{i}(x), f_{i}^{\prime}(x) \in G_{\delta_{i}}^{\prime}(i=\overline{1, s})$. Since $f_{i}(x), f_{i}^{\prime}(x) \in G_{\delta_{i}}^{\prime} \subseteq G^{\prime}$ and $G^{\prime}$ is extragraded, by $v i)$ it follows that $\delta\left(f_{i}(x)^{-1} f_{i}^{\prime}(x)\right)<\delta_{i}$ and hence $f_{i}^{-1} f_{i}^{\prime} \in$ $\operatorname{hom}\left(G, G^{\prime}\right)_{\delta_{i}}(i=\overline{1, s})$.

We have proven so far that six axioms of extragraduation are satisfied, hence $\operatorname{HOM}\left(G, G^{\prime}\right)$ is an extragraded group, but since every extragraduation is a paragraduation, as is stated in Theorem $1.4, \operatorname{HOM}\left(G, G^{\prime}\right)$ is a paragraded group.

b) It is easy to establish that $\operatorname{HOM}\left(M, M^{\prime}\right)$ is a commutative group. This group is a paragraded group, since $\operatorname{HOM}\left(M, M^{\prime}\right)$ is a homogeneous subgroup of $\operatorname{hom}\left(M, M^{\prime}\right)$, and $\operatorname{hom}\left(M, M^{\prime}\right)$ is a paragraded group according to [7].

Remark 2.7. From the last proof we notice that $\operatorname{HOM}\left(G, G^{\prime}\right)$ is a postparagraded group if $G, G^{\prime} \in G_{\Delta}^{P}$.

Remark 2.8. In the same way that we defined the categories $G_{\Delta}^{P}$ and $M_{\Delta}^{P}$, we define the categories of extragraded groups and modules with the set of grades $\Delta$ and denote it by $G_{\Delta}^{E}$ and $M_{\Delta}^{E}$, respectively.

In what follows, all objects are assumed to be commutative.

Let us now observe the map $\operatorname{HOM}\left(G_{1},-\right): G_{\Delta}^{E} \rightarrow G_{\Delta}^{P}$ which sends each $G_{2} \in G_{\Delta}^{E}$ to $\operatorname{HOM}\left(G_{1}, G_{2}\right) \in G_{\Delta}^{P}$ and each $g: G_{2} \rightarrow G_{2}^{\prime}$ from $\operatorname{hom}_{G_{\Delta}^{P}}\left(G_{2}, G_{2}^{\prime}\right)$, where $G_{2}, G_{2}^{\prime} \in G_{\Delta}^{E}$, to a morphism

$$
\operatorname{HOM}\left(1_{G_{1}}, g\right): \operatorname{HOM}\left(G_{1}, G_{2}\right) \rightarrow \operatorname{HOM}\left(G_{1}, G_{2}^{\prime}\right)
$$


defined as follows. For each $x \in \operatorname{HOM}\left(G_{1}, G_{2}\right)$ we have

$$
x=\left\langle f_{\delta} \mid \delta \in \Delta\right\rangle,
$$

where $f_{\delta} \in \operatorname{hom}\left(G_{1}, G_{2}\right)_{\delta}$. So, let

$$
\operatorname{HOM}\left(1_{G_{1}}, g\right)(x)=\left\langle g \circ f_{\delta} \mid \delta \in \Delta\right\rangle .
$$

Then $\operatorname{HOM}\left(G_{1},-\right)$ represents a covariant functor. First,

$$
\operatorname{HOM}\left(1_{G_{1}}, 1_{G_{2}}\right)(x)=\left\langle f_{\delta} \mid \delta \in \Delta\right\rangle=x .
$$

Now, let $g_{1}, g_{2} \in \operatorname{hom}_{G_{\Delta}^{P}}\left(G_{2}, G_{2}^{\prime}\right)$ and let $g_{1}, g_{2}$ be composable. Then we have

$$
\begin{aligned}
\operatorname{HOM}\left(1_{G_{1}}, g_{1}\right) \circ \operatorname{HOM}\left(1_{G_{1}}, g_{2}\right)(x) & =\operatorname{HOM}\left(1_{G_{1}}, g_{1}\right)\left\langle g_{2} \circ f_{\delta} \mid \delta \in \Delta\right\rangle \\
& =\left\langle g_{1} \circ g_{2} \circ f_{\delta} \mid \delta \in \Delta\right\rangle \\
& =\operatorname{HOM}\left(1_{G_{1}}, g_{1} \circ g_{2}\right)(x),
\end{aligned}
$$

for all $x \in \operatorname{HOM}\left(G_{1}, G_{2}\right)$.

Similarly, observe the map $\operatorname{HOM}\left(-, G_{2}\right): G_{\Delta}^{P \text { op }} \rightarrow G_{\Delta}^{P}$ which sends each $G_{1} \in G_{\Delta}^{P^{\text {op }}}$ to $\operatorname{HOM}\left(G_{1}, G_{2}\right) \in G_{\Delta}^{P}$ and each $f: G_{1} \rightarrow G_{1}^{\prime}$ from $\operatorname{hom}_{G_{\Delta}^{P}}\left(G_{1}, G_{1}^{\prime}\right)$ to a morphism

$$
\operatorname{HOM}\left(f, 1_{G_{2}}\right): \operatorname{HOM}\left(G_{1}, G_{2}\right) \rightarrow \operatorname{HOM}\left(G_{1}^{\prime}, G_{2}\right)
$$

defined by

$$
\operatorname{HOM}\left(f, 1_{G_{2}}\right)(x)=\left\langle f_{\delta} \circ f \mid \delta \in \Delta\right\rangle,
$$

for all $x=\left\langle f_{\delta} \mid \delta \in \Delta\right\rangle \in \operatorname{HOM}\left(G_{1}, G_{2}\right)$. The proof that $\operatorname{HOM}\left(-, G_{2}\right)$ is a contravariant functor is similar to the one that $\operatorname{HOM}\left(G_{1},-\right)$ is a covariant functor and we shall omit it. Also, it is easy to verify that the following diagram commutes

$$
\begin{gathered}
\operatorname{HOM}\left(G_{1}, G_{2}\right) \stackrel{\operatorname{HOM}\left(1_{G_{1}}, g\right)}{\longrightarrow} \operatorname{HOM}\left(G_{1}, G_{2}^{\prime}\right) \\
\operatorname{HOM}\left(f, 1_{G_{2}}\right) \downarrow \\
\operatorname{HOM}\left(G_{1}^{\prime}, G_{2}\right) \underset{\operatorname{HOM}\left(1_{G_{1}^{\prime}}, g\right)}{\longrightarrow \operatorname{HOM}(f, g)} \operatorname{HOM}\left(G_{1}^{\prime}, G_{2}^{\prime}\right)
\end{gathered}
$$

Thus, $\operatorname{HOM}(-,-): G_{\Delta}^{P \text { op }} \times G_{\Delta}^{E} \rightarrow G_{\Delta}^{P}$ is a bifunctor. Let us fix $G_{1} \in G_{\Delta}^{P}$ and observe the functor $\operatorname{HOM}\left(G_{1},-\right): G_{\Delta}^{E} \rightarrow G_{\Delta}^{P}$. Suppose that the following 
sequence

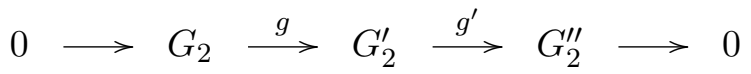

is exact, where $G_{2}, G_{2}^{\prime}, G_{2}^{\prime \prime} \in G_{\Delta}^{E}$. If we apply the functor $\operatorname{HOM}\left(G_{1},-\right)$ to the sequence (2.4), we wish to prove that the sequence

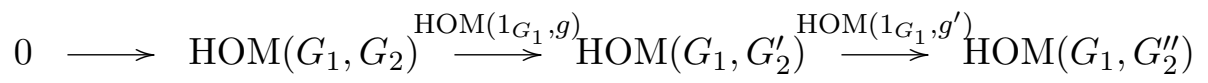

is exact. Let $x=\left\langle f_{\delta} \mid \delta \in \Delta\right\rangle \in \operatorname{ker} \operatorname{HOM}\left(1_{G_{1}}, g\right)$. Then $\operatorname{HOM}\left(1_{G_{1}}, g\right)(x)=$ $\left\langle g \circ f_{\delta} \mid \delta \in \Delta\right\rangle$. From $x \in \operatorname{ker} \operatorname{HOM}\left(1_{G_{1}}, g\right)$ and the exactness of the sequence (2.4), it follows that $x=f_{0}$ which proves the injectivity of $\operatorname{HOM}\left(G_{1},-\right)$. If $y \in \operatorname{im} \operatorname{HOM}\left(1_{G_{1}}, g\right)$, then $y=\left\langle g \circ f_{\delta} \mid \delta \in \Delta\right\rangle$ for some $\left\langle f_{\delta} \mid \delta \in \Delta\right\rangle \in$ $\operatorname{HOM}\left(G_{1}, G_{2}\right)$. By exactness of $(2.4)$, im $g \subseteq \operatorname{ker} g^{\prime}$, so

$$
\operatorname{HOM}\left(1_{G_{1}}, g^{\prime}\right)(y)=\left\langle g^{\prime} \circ g \circ f_{\delta} \mid \delta \in \Delta\right\rangle=0 .
$$

Hence, $y \in \operatorname{ker} \operatorname{HOM}\left(1_{G_{1}}, g^{\prime}\right)$. Now, if $y \in \operatorname{ker} \operatorname{HOM}\left(1_{G_{1}}, g^{\prime}\right)$, then if $a \in G_{1}$, we have $y(a)=\left\langle f_{\delta}^{\prime}(a) \mid \delta \in \Delta\right\rangle$, where $f_{\delta}^{\prime} \in \operatorname{hom}\left(G_{1}, G_{2}^{\prime}\right)_{\delta}$. Note that $f_{\delta}^{\prime}(a) \in \operatorname{ker} g^{\prime}=\operatorname{im} g$, for all $\delta \in \Delta$, so $f_{\delta}^{\prime}(a)=g(b)$ for some $b=f_{\delta}(a) \in G_{2}$, where $f_{\delta} \in \operatorname{hom}\left(G_{1}, G_{2}\right)_{\delta}$. This proves that $y \in \operatorname{im} \operatorname{HOM}\left(1_{G_{1}}, g\right)$. Thus, $\operatorname{HOM}\left(G_{1},-\right): G_{\Delta}^{P} \rightarrow G_{\Delta}^{P}$ is a left exact functor. Similarly, one can prove that $\operatorname{HOM}\left(-, G_{2}\right): G_{\Delta}^{P^{\text {op }}} \rightarrow G_{\Delta}^{P}$ is also a left exact functor. This means that the following proposition holds.

Proposition 2.9. A bifunctor $\operatorname{HOM}(-,-): G_{\Delta}^{P \mathrm{op}} \times A b G_{\Delta}^{E} \rightarrow A b G_{\Delta}^{P}$ is left exact.

Analogously, one can prove that the following proposition holds as well.

Proposition 2.10. A bifunctor $\operatorname{HOM}(-,-): M_{\Delta}^{P \mathrm{op}} \times M_{\Delta}^{P} \rightarrow A b G_{\Delta}^{P}$ is left exact.

Lemma 2.11. Let $M$ be a paragraded $R$-module with the set of grades $\Delta$. Then there exists a free paragraded $R$-module.

Proof. Let $M^{\prime}$ be a free $R$-module with base $M$. If we define the map $\pi^{\prime}$ : $\Delta \rightarrow \operatorname{Sg}\left(M^{\prime},+\right)$ via $\pi^{\prime}(\delta)=M_{\delta}^{\prime}$, where $M_{\delta}^{\prime}$ denotes the subgroup generated by the set $M_{\delta}$, then it can be proven that $\pi^{\prime}$ is paragraduation of $M^{\prime}$.

Corollary 2.12. The category $M_{\Delta}^{P}$ has enough injective and projective objects.

The proofs of the following propositions are similar to the proofs in the case of abstract modules.

Proposition 2.13. Let $M$ be a paragraded left $R$-module of type $\Delta$. Then: 
a) $M$ is projective iff the functor $\operatorname{HOM}(M,-)$ is exact;

b) $M$ is injective iff the functor $\operatorname{HOM}(-, M)$ is exact.

Proposition 2.14. Let $M^{\prime}$ be a projective paragraded left $R$-module of type $\Delta$ and $\bar{M}$ an injective paragraded left $R$-module of type $\Delta$. Then:

a) $\operatorname{EXT}^{n}\left(M^{\prime}, M\right)=\{0\}$, for all $n \geq 1$ and for every paragraded left $R$-module $M$ of type $\Delta$;

b) $\operatorname{EXT}^{n}(M, \bar{M})=\{0\}$, for all $n \geq 1$ and for every paragraded left $R$-module $M$ of type $\Delta$;

where EXT designates the right derived functor of HOM.

\section{REFERENCES}

[1] S. Awodey, Category Theory, Clarendon Press, Oxford 2006.

[2] N. Bourbaki, Algèbre, Chap. II, 3e édit. Paris, Hermann, 1962.

[3] E. Ilić-Georgijević, Some problems from the theory of paragraded structures, $\mathrm{Ph} . \mathrm{D}$. Thesis, University of East Sarajevo, 2011.

[4] M. Krasner and M. Vuković: Structures paragraduées (groupes, anneaux, modules) I, Proc. Japan Acad., 62 (1986), Ser, A, No. 9, 350-352.

[5] M. Krasner and M. Vuković, Structures paragraduées (groupes, anneaux, modules) II, Proc. Japan Acad., 62 (1986), Ser, A, No. 10, 389-391.

[6] M. Krasner and M. Vuković, Structures paragraduées (groupes, anneaux, modules) III, Proc. Japan Acad., 63 (1987), Ser, A, No. 1, 10-12.

[7] M. Krasner and M. Vuković, Structures paragraduées (groupes, anneaux, modules), Queen's Papers in Pure and Applied Mathematics, No. 77, Queen's University, Kingston, Ontario, Canada 1987, pp. 163.

[8] S. Mac Lane, Categories for the Working Mathematician, Springer-Verlag New York, 2nd ed. 1998.

[9] C. Năstăsescu and F. Van Oystaeyen, Methods of Graded Rings, Springer-Verlag, Berlin Heidelberg 2004.

[10] V. Perić, Algebra I, IP Svjetlost, Sarajevo, 1991.

[11] J. J. Rotman, An Introduction to Homological Algebra, Springer, 2nd ed. 2009.

[12] M. Vuković, Structures graduées et paragraduées, Prepublication de l'Institut Fourier, Université de Grenoble I, No. 536 (2001), 1-40.

[13] M. Vuković, About Krasner's and Vuković's paragraduations, International Congress of Mathematicians, Hyderabad, India, 19-27 August 2010.

(Received: September 9, 2011)

(Revised: October 9, 2011)
Faculty of Civil Engineering

University of Sarajevo

E-mail: emil.ilic.georgijevic@gmail.com 